\title{
Multi-Agent Architecture for Implementation of ITIL Processes: Case of Incident Management Process
}

\author{
Youssef SEKHARA, Hicham MEDROMI, Adil SAYOUTI \\ Equipe Architectures des Systèmes (EAS), Laboratoire d'Informatique, Systèmes et Energies Renouvelables (LISER) \\ Hassan II University-Aïn Chock, Ecole Nationale Supérieure d'Electricité et de Mécanique (ENSEM) \\ Casablanca, Morocco
}

\begin{abstract}
ITIL (Information Technology Infrastructure Library) is the most widely accepted approach to IT service management in the world. Upon the adoption of ITIL processes, organizations face many challenges that can lead to increased complexity. In this paper we use the advantages of agent technology to make implementation and use of ITIL processes more efficient, starting by the incident management process.
\end{abstract}

Keywords-ITIL; Process; Multi-agent system; Incident Management Process

\section{INTRODUCTION}

The use of computers has become essential for business organizations. Having the best technology will not guarantee the expected needed service reliability. It is necessary for them to have a full service around these technologies. There is a large number of repositories that reflect the best practices developed over the years, ITIL presents a guide of best applicable practices suitable to all types of organizations providing services to a business organization [1]. ITIL shows the framework of the organization, objectives, processes related to major activities of information services and their interactions. It is a kind of canvas that can be used by the directions of information systems to design their own organization.

The successful adoption of ITIL processes is a major challenge for many managers of information systems. Agent technology has shown great potential for solving problems especially for complex applications involving interaction between several entities. Our job is to use the advantages of agent technology to make more efficient implementation and use of ITIL processes.

In this paper, characteristics and the importance of ITIL in information systems are presented in the second section. The third section gives a view on problems encountered in the implementation of ITIL and the contribution of multi-agent systems to make it easier and more efficient. The fourth section shows the multi agent architecture proposed to implement incident management process chosen to be the starting point for the implementation. In the fifth section, we describe the implementation.

\section{ITIL \& MULTI-AGENT SySTEM}

\section{A. Presentation of ITIL}

The IT Service Management ITIL is based on five groups of activities (each containing its multiple processes) to manage the service throughout its life cycle [2]; ITIL recommends taking into account management of services, from the phases of study and defining the needs of IT projects. This, provides several benefits to all teams of the production or development, but serves primarily to provide a more reliable service to the customer, it also validates that we have many resources and skills necessary for the operation of this new application, which involves taking into account the impact on physical infrastructure in areas such as capacity, performance, availability, reliability and maintenance.

The five phases are[3]:

- Service Strategy : The phase of strategic planning of service management capabilities, and the alignment of service and business strategies.

- Service Design : The phase of designing and developing appropriate IT services, including architecture, processes, policy and documents; the design goal is to meet the current and future business requirements

- Service Transition: The phase of realizing the requirements from previous stages, and improving the capabilities for the transition of new and modified services to production

- Service Operation : The phase of achieving effectiveness and efficiency in providing and supporting services in order to ensure value for the customer and the service provider.

- Continual Service Improvement: The phase of creating and maintaining the value for the customer by design improvement, and service introduction and operation

In the ITIL framework, the quality of service is based on a structure of measurable possible repeated activities in interrelated processes. This approach to service management by process is now recognized as the most effective by a large number of companies. The implementation of processes providing efficient operation of these systems is therefore an important element to enable businesses to take full advantage of their entire IT infrastructure.

A process consists of several activities generating results to clients. It is called after a trigger event. A well-studied process must achieve the objectives, using optimal time, money and 
resources. A process is provided by roles and not directly by the people, what makes this notion generic and independent of the organization.

\section{B. Challenges of ITIL adoption \& Proposition of multi-agent solution}

Despite the flexibility of ITIL and its processes, organizations are faced with many challenges when adopting ITIL best practices that can lead to increased complexity. The inability to control this complexity leads to increased costs and longer time lines for implementation. Such as:

- Lack of management commitment: it presents the main cause of the failure of the implementation of ITIL because without it nothing tends to occur.

- Choosing an inefficient point of departure: for the adoption of ITIL there is not a standard starting point, every business must start with a gap analysis between existing and ITIL benefits to be able to find areas where organization will receive more value by adopting ITIL.

- Resistance to ITIL: ITIL is a major organizational change which is always resisted. Departments, teams and individuals tend to defend the current status.

- The close relationship between activities of different processes.

Faced with these problems, the leaders of IT departments have recourse to incorporate ITIL's compliant software permitting to accompany and make the implementation of ITIL processes eaiser[4].

There are several free and commercial service software for the automation of repetitive tasks and reducing human error and cost, but these are just tools that help to meet the objectives as explained by Claude Durant, founder of itSMF France (The IT Service Management Forum France): they are only supports, prior to use, you must define and implement objectives of ITIL processes such as incident management and problem management for example. The tool will then help to meet the set objectives of performance and quality [5].

We propose to design and implement a multi-agent distributed platform that implements the various ITIL process through autonomous entities (agents) that interact with each other and share the knowledge, reasoning, and decision, that leads to profit from the benefits of multi-agent systems to facilitate the adoption of ITIL and fully enjoy the process.

Distribution using multi-agent systems is very useful given the nature of the problem addressed and the complexity of the system permitting the implementation of the various tasks of ITIL processes; we may explain the need for distributing by multi-agent systems for the following reasons:

- Complexity of the problem: ITIL processes involve many subsystems very diverse in nature, with many features, interacting with several human experts (operators, experts, technicians, etc..), who are often distributed in the physical space.

- Need for local vision: the solutions based on local approaches such as agent approach often allow to fastly solve the problems especially those are too large to be analyzed globally.

- Adaptation to changes in the structure or environment: the multi-agent systems, thanks to their distributed nature -because they always involve a local reasoning- allow the integration and the appearance or disappearance of agents during operation, which enables to build suitable architectures to consider scalability and adaptation needed to operate the system

\section{Definition \& advantages of multi-agent systems}

Multi-agent systems (MAS) are systems where multiple agents can interact with each other in various modes including cooperation, competition or mutual existence[6].

An agent is a hardware or (more usually) a software-based computer system that enjoys the following properties[7]:

- Autonomy : agents operate without the direct intervention of humans or others, and have some kind of control over their actions and internal state;

- Social ability : agents interact with other agents (and possibly humans) via some kind of agentcommunication language;

- Reactivity: agents perceive their environment and respond in a timely fashion to changes that occur in it;

- Pro-activeness: agents do not simply act in response to their environment; they are able to exhibit goal-directed behaviour by taking initiative.

The need for autonomy makes the agent support activities in an intelligent and flexible way and can adapt to the environment without requiring human intervention [8].

The main characteristics of a multi-agent system are:

- Group of agents acting and working independently of each other,

- Each agent is a part of the system

- Each agent works to accomplish its tasks, each agent in the proposed architecture allows to perform the tasks related to the concerned process

- Each agent is able to communicate and interact with other agents, which allows a rich exchange between different processes

- An agent is able to coordinate its activities with other agents to access shared resources

- Agents' common goal in our architecture is the proper functioning of the process

- Each agent has a partial view of the MAS.

Some advantages of using multi-agent systems [9] compared to other technologies:

- Scalability and flexibility; it is easy to add new agents to the system. 
- The development and reusability, since it is easier to develop and maintain a modular software

\section{- Robustness and reliability}

\section{INCIDENT MANAGEMENT PROCESS}

\section{A. Approach of implementation}

ITIL has many processes. Each ITIL process has a great impact on the organization. it is preferable that the full implementation of ITIL is spread over several phases. A big bang ITIL has the potential to disrupt the SI as well as commercial operations [10]. This leads us to choose the bottom-up approach for implementing process relying on the scalability and flexibility of multi-agent systems; we therefore start by the implementation of each process in order to integrate all in one platform.

We chose to start with the implementation of the incident management process. Whatever the quality of the information system set up in the company or the skills of technicians who operate, incidents occur. These incidents always have an important effect on the trust that users place in the team who manages this information system. How to handle these "crises" and their rapidity of resolution is an indicator of the maturity of the IT team. Incident Management has strong links with the management problem for the identification of causes, and change management for the implementation of changes after identification of causes. There are also links with configuration management [11]. That is why starting with the implementation of the incident management process is particularly important.

\section{B. Incident Management process}

Incident Management Process is responsible for all steps from the detection and recording of an incident until it is resolved and closed. The aim is to restore service as quick as possible with minimal disruption to the business.

In ITIL terminology [12], an 'incident' is defined as: An unplanned interruption to an IT service or reduction in the quality of an IT service. Failure of a configuration item that has not impacted service yet is also an incident, for example failure of one disk from a mirror set.

Incidents can be triggered in several ways. The most common way is when a user calls the service center or completes an online form of the incident in a tool or via the Internet. However, many incidents are recorded by event management tools.
The incident management process consists of the following steps [13]:

1) Identification : The incident is detected or reported.

2) Registration : An incident record is created.

3) Categorization : The incident is coded by type, status, impact, urgency, SLA, et cetera.

4) Prioritization: Every incident gets an appropriate prioritization code to determine how the incident is handled by support tools and support staff.

5) Diagnosis : A diagnose is carried out to try to discover the full symptoms of the incident.

6) Escalation: When the service desk cannot resolve the incident itself, the incident is escalated for further support (functional escalation). If incidents are more serious, the appropriate IT managers must be notified (hierarchic escalation).

7) Investigation and diagnosis : If there is no known solution, THE INCIDENT IS INVESTIGATED.

8) Resolution and recovery: Once the solution has been found, the issue can be resolved.

9) Incident closure : The service desk should check that the incident is fully resolved and that the user is satisfied with the solution and the incident can be closed

\section{PROPOSED IMPROV}

\section{A. Proposed architecture}

Our architecture (Figure 1) consists of two layers: a reactive layer and a deliberative layer. The reactive layer consists of reactive agents (simple processing units that perceive and react to changes in their environment [14]), in our architecture the User Agent is reactive agent, The deliberative layer is composed of other agents that are cognitive agents (in which decision making depends upon the manipulation of data structures representing the beliefs, desires, and intentions of the agent [15]).The communication between agents is done using messages.

\section{B. Description of the architecture}

Figure 2 presents the sequence diagram which gives an overview on the different interactions between agents in our architecture in order to better understand its behaviour [16]. After reporting the incident to User Agent linked to an interface, it sends the incident's information to the Diagnosis agent, which records it in the incident database and determines whether the incident has already occurred. 


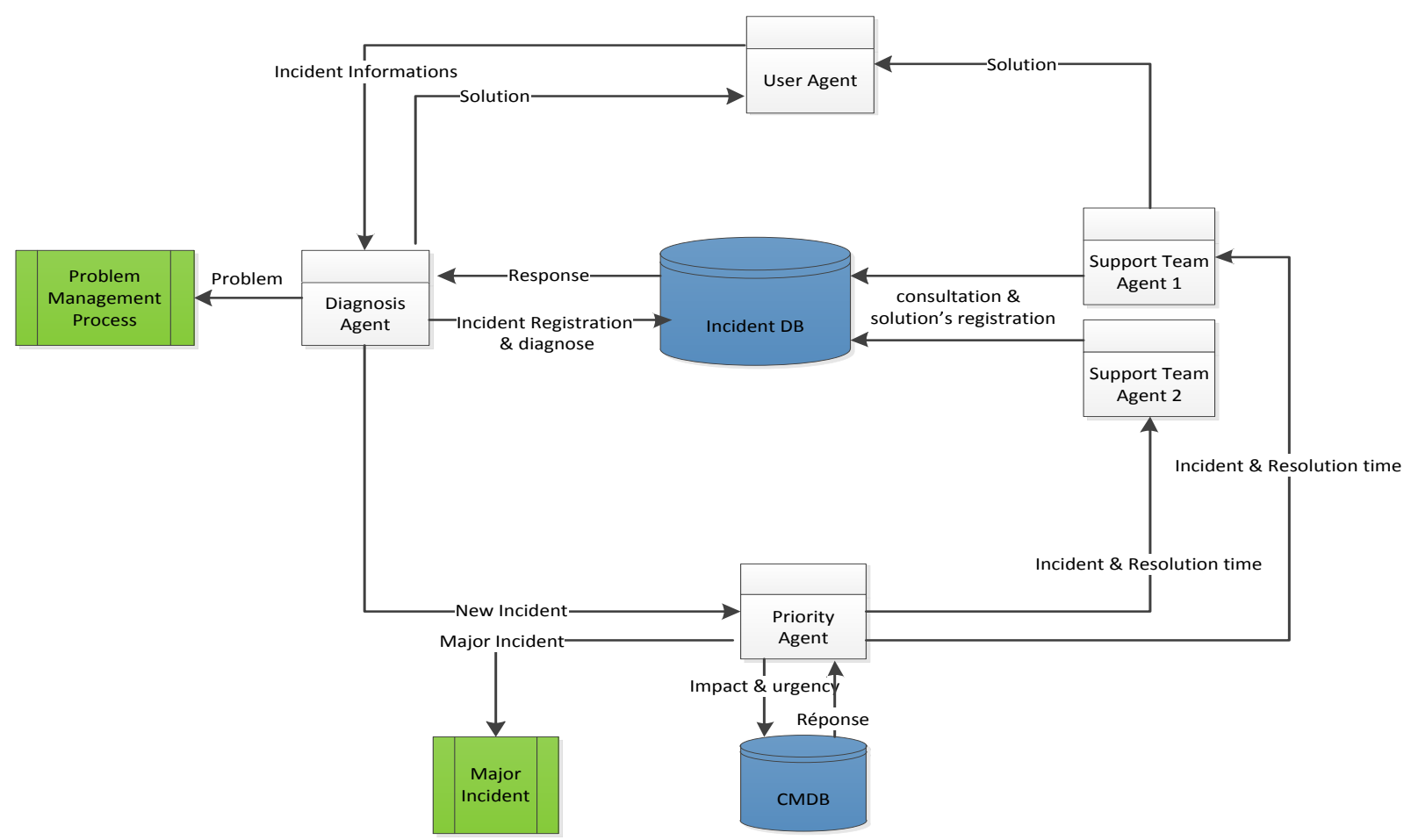

Fig. 1. Architecture of incident management process implementation

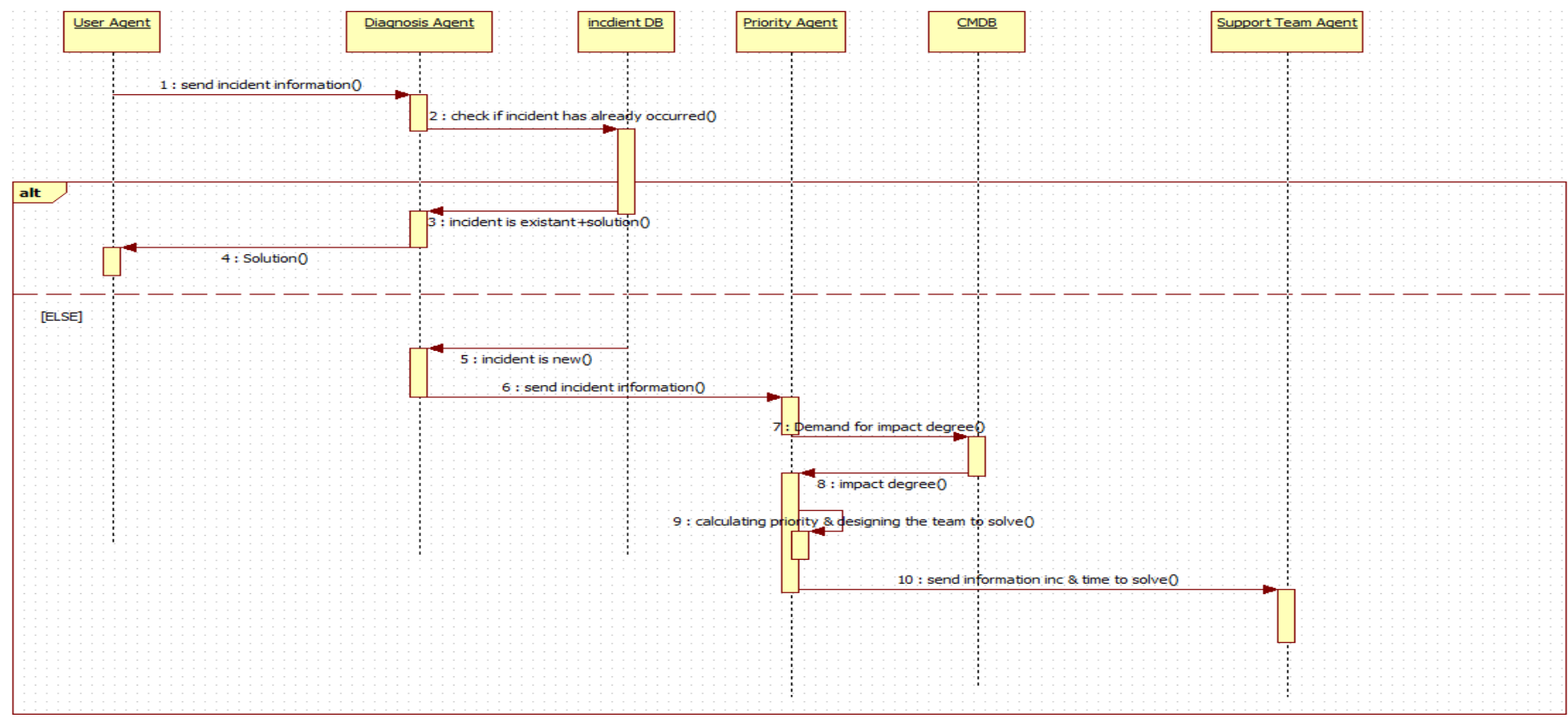

Fig. 2. Sequence diagram of the architecture

In this case, it returns to the user the solution present in the database, this operation saves considerable time. The Diagnosis Agent calculates the rate of production of this incident and in case it exceeds a predetermined value in the service level agreement (SLA) the Diagnosis Agent shall forward the incident to the responsible of problem management process to find the source of the problem.

In the case where the incident occurs for the first time, the Diagnosis Agent sends incident's information to the Priority
Agent that calculates the priority of the incident according to the degree of impact and urgency presents in the configuration management database (CMDB).

This calculation is used to define the time allocated to the resolution and this is communicated to the appropriate Support Team Agent according to the type of the incident (for example if the incident concerns the software, the incident and resolution time presented to the team that deals with incidents softwares). If the problem has a very high degree of priority, 
the Priority Agent shall communicate it to the team that deals with major incidents. Once the solution is found, it's recorded by the Support Team Agent in the incident database and sent to the user.

\section{APPLICATION}

We implemented this architecture using Java language and Java Message Service (JMS) using Apache ActiveMQ language to develop the different agents and also their interactions. Figure 3 presents the user interface where he puts information about the incident who wanted to be solved.

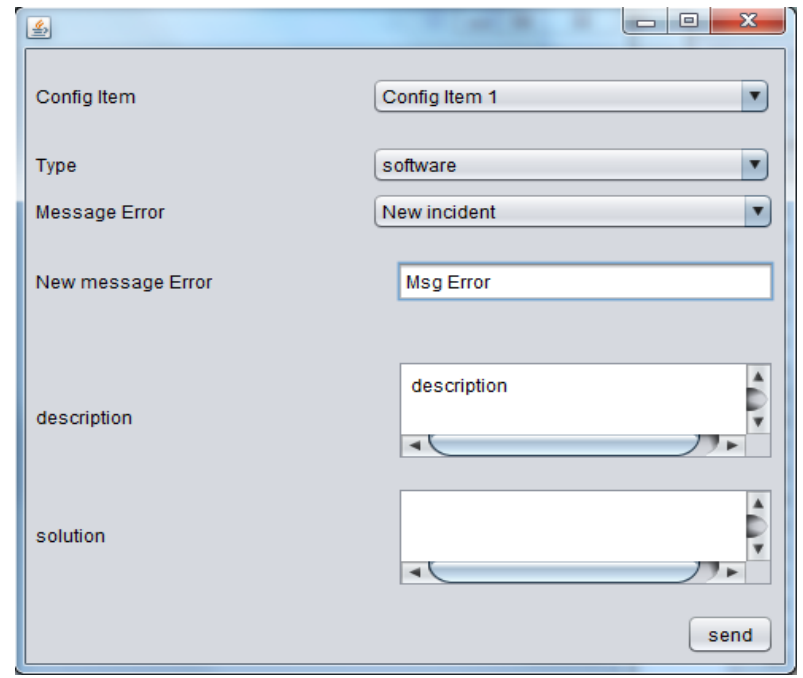

Fig. 3. User interface

\section{CONCLUSIONS}

The first part of this paper presented characteristics and the importance of ITIL in information systems. The second part showed problems encountered in the implementation of ITIL and the contribution of multi-agent systems to make it easier and more efficient. In the third part we talked about the choice of incident management process as a starting point for the implementation by the agents. In the last part, we proposed multi agent architecture to implement incident management process.
We started with the process of incident management, chosen to be the core of this architecture because it has strong links with other processes such as process problem management, availability management and continuity management that we aim to develop in the following step and merge them into a complete system

\section{REFERENCES}

[1] Y. Sekhara, H. Medromi and A. Sayouti, " Systmème multi-agent pour l'implementation du processus de gestion d'incidents ITIL", in Proc. WOTIC'13, 2013.

[2] itSMF France "ITIL : Information Technology Infrastructure Library". [Online]. Available: http://www.itilfrance.com

[3] A. de Jong, A. Kolthof, M. Pieper, R. Tjassing, A. van der Veen, T. Verheijen, "ITIL $®$ V3 Foundation Exam - The Study Guide" pp. 23-26.

[4] Y. Sekhara, H. Medromi and A. Sayouti, "Etude des logiciels conformes à ITIL et proposition d'une Solution Distribuée à base multi-agent pour la gestion de la CMDB au Coeur des Processus ITIL", in Proc. JDTIC'12, 2012.

[5] INDEXEL. "Les logiciels Itil, indispensables mais pas autosuffisants". [Online]. Available: http://www.indexel.net/materiels/les-logiciels-itilindispensables-mais-pas-autosuffisants.html

[6] J. Ferber, "Les Systèmes Multi Agents: vers une intelligence". InterEditionsBradshaw 1995. pp. 28.

[7] M. Wooldridge, and N. R. Jennings, "Agent theories, architectures, and languages". In Wooldridge and Jennings, eds. Intelligent Agents, Springer Verlag, 1995. pp. 1-22.

[8] N. R. Jennings, M. Wooldridge, "Agent technology: foundation, application, and markets". Springer, NewYork 1998.

[9] Katia P. Sycara, "Multiagent Systems" the American Association for Artificial Intelligence 1998.

[10] BMC software, "ITIL pour les PME/PMI". [Online]. Available: www.bmc.com/fr-CA/documentation

[11] C. Dumont. "ITIL Pour un service informatique optimal" Eyerolles. pp. 87.

[12] Office of Government Commerce, "ITIL V3 - Service Operation". pp. 86.

[13] A. de Jong, A. Kolthof, M. Pieper, R. Tjassing, A. van der Veen, T. Verheijen, "ITIL ® V3 Foundation Exam - The Study Guide" pp. 90.

[14] Adina Magda Florea, "Introduction to Multi-Agent Systems". International Summer School on Multi-Agent Systems, Bucharest, 1998.

[15] M. Wooldridge, "Intelligent Agents", Multiagent Systems:A Modern Approach to Distributed Modern Approach to Artificial Intelligence, edited by Gerhard Weiss. Massachusetts Institute of Technology1999. pp. 27-79.

[16] X. Blanc, I. Mounier. "UML2 pour les développeurs". pp. 64-77. 